\title{
Toward Single Electron Resolution Phonon Mediated Ionization Detectors
}

\author{
Nader Mirabolfathi ${ }^{\mathrm{a}}$, H. Rusty Harris ${ }^{\mathrm{a}}$, Rupak Mahapatra ${ }^{\mathrm{a}}$, Kyle Sundqvist ${ }^{\mathrm{a}}$, Andrew Jastram ${ }^{\mathrm{a}}$, Bruno \\ Serfass $^{\mathrm{b}}$, Dana Faiez ${ }^{\mathrm{b}}$, Bernard Sadoulet ${ }^{\mathrm{b}}$ \\ ${ }^{a}$ Department of Physics and Astronomy, Texas A $8 M$ University \\ ${ }^{b}$ Department of Physics, University of California at Berkeley
}

\begin{abstract}
Experiments seeking to detect rare event interactions such as dark matter or coherent elastic neutrino nucleus scattering are striving for large mass detectors with very low detection threshold. Using the NeganovLuke phonon amplification effect, the Cryogenic Dark Matter Search (CDMS) experiment is reaching unprecedented RMS resolutions of $\sim 14 \mathrm{eV}_{e e}$. CDMSlite is currently the most sensitive experiment to WIMPs of mass $\sim 5 \mathrm{GeV} / \mathrm{c}^{2}$ but is limited in achieving higher phonon gains due to an early onset of leakage current into the Germanium crystal detectors. Because the contact interface geometry is particularly weak for blocking hole injection from the metal, a new design is demonstrated which allows a high voltage bias via a vacuum separated electrode. With an increased bias voltage and subsequent factor of two increase in Luke phonon gain, a world best RMS resolution at $\mathrm{E}=0 \mathrm{eV}$ of sigma $\sim 7 \mathrm{eV}_{e e}$ for $0.25 \mathrm{~kg}(\mathrm{~d}=75 \mathrm{~mm}, \mathrm{~h}=1 \mathrm{~cm})$ Ge detectors was achieved. Since the leakage current is a function of the electric field and the phonon gain is a function of the applied voltage, appropriately robust interface blocking material combined with thicker substrate $(25 \mathrm{~mm})$ will reach a resolution of $\sim 2.8 \mathrm{eV}_{e e}$. In order to achieve a resolution of $\sim 1 \mathrm{eV}$, we are investigating a layer of insulator between the phonon readout surface and the semiconductor crystals.
\end{abstract}

\section{Motivation: Ultra low threshold detec- tors for Dark Matter and Coherent Neu- trino scattering detection}

A large body of astrophysical observations suggest that $85 \%$ of the matter in the universe is not made of known standard model particles [1]. Understanding the nature of this dark matter is of fundamental importance to cosmology, astrophysics, and high energy particle physics. Although Weakly Interacting Massive Particles (WIMPs) of mass 10$100 \mathrm{GeV} / c^{2}$ have been the main interest of the majority of direct dark matter detection experiments, recent claims for signal, together with compelling new theoretical models, are shifting the old paradigm toward broader regions well below mass of $10 \mathrm{GeV} / c^{2}[2]$.

Very low energy recoils and small interaction rates from such low mass WIMPs are expected, thus large mass detectors with very low threshold are highly desired. These very low threshold detectors are sine qua non for any attempt to detect very light mass $\left(<1 \mathrm{GeV} / c^{2}\right)$ dark matter. They are also a necessary requisite for observing coherent elastic

Preprint submitted to Nuclear Instruments and Methods A neutrino-nucleus scattering [3], a standard model process that has recently been proposed as a sensitive and flavor invariant probe for sterile neutrinos, neutrino magnetic moment and other high energy physics effects $[4,5]$.

P. Luke had suggested to utilize very low noise readout designed for phonon mediated detectors to indirectly measure ionization in semiconductor detectors [6]. The measurement principle is based on the fact that carriers drifting through crystals under an applied electric field release phonons whose total energy is proportional to the interaction energy as well as the applied bias voltage as

$$
E_{\text {Luke }}=V_{\text {bias }} E / \epsilon,
$$

where $E$ is the energy of the interaction and $\epsilon$ is the average energy necessary to produce electron and hole pairs. Since the total signal is proportional to the bias potential, in the absence of any leakage current the Signal-to-Noise Ratio (SNR) improves proportional to the bias and can be improved down to single electron-hole sensitivity. CDMSlite is using this very sensitive method to search for low mass dark matter and is currently the most sensitive ex-

February 3, 2017 
periment for WIMPs of masses $<5 \mathrm{GeV} / c^{2}$ [7]. However, the current generation of CDMSlite detectors exhibit a leakage current for fields as low as $24 \mathrm{~V} / \mathrm{cm}$, thus single ionization sensitivity has not yet been realized. This early onset of leakage is in contrast with results from standard $77 \mathrm{~K}$ depleted Ge detectors which are usually operated with much larger fields $(\sim 1000 \mathrm{~V} / \mathrm{cm})$. Recent understanding of the CDMSlite interface shows that it is comprised of polycrystalline grain boundaries which allow charge leakage. Here we report on our recent studies and success toward understanding this early onset of leakage current and our suggestions to improve ionization contacts for the ultimate single electron resolution detectors.

\section{Bulk breakdown versus carrier injection through contact}

We can think of three sources of excessive leakage current in CDMS detectors: crystal break down, carrier injection through metal/germanium contact or conduction on non passivated free surfaces of a detector. The surface conduction possibility is eliminated outright because of precise, clean crystal fabrication and handling. Furthermore, the amount of current leakage observed cannot be accounted for in surface current density without significant damage to the detector.

\subsection{Ge Crystal bulk breakdown}

The first evidence against bulk crystal breakdown is the fact that such breakdown is catastrophic and irreversible. We observe that reverse biased detectors that have previously experienced significant leakage do not demonstrate high leakage when appropriately biased. The second set of evidence is the low electric field $\sim 30 \mathrm{~V} / \mathrm{cm}$ at which high leakage is observed.

In a previous study we ruled out breakdown in high purity Ge crystals by operating a Majorana prototype P-type Point Contact Ultra pure Ge detector (PPC) in a similar setup in which we operate CDMS detectors [8]. A 17 g PPC prototype was equipped with a tungsten Transition Edge Sensor (TES) thermistor with both ionization and phonon measured up to $400 \mathrm{~V}$ as shown in Fig. 1. There were no sign of crystal breakdown despite large fields present in the vicinity of the point contact (up to $7000 \mathrm{~V} / \mathrm{cm}$ ). This clearly ruled out crystal breakdown. We also verified that the phonon gain
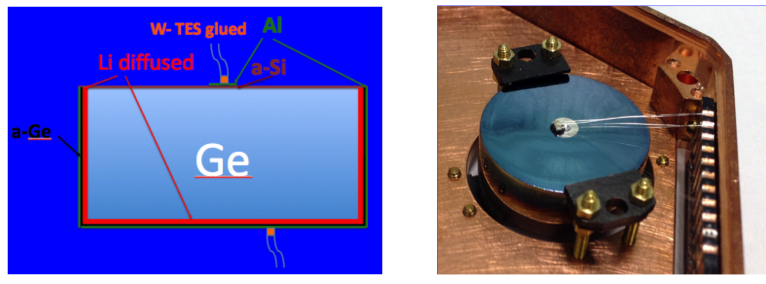

Ionization v.s. Phonon

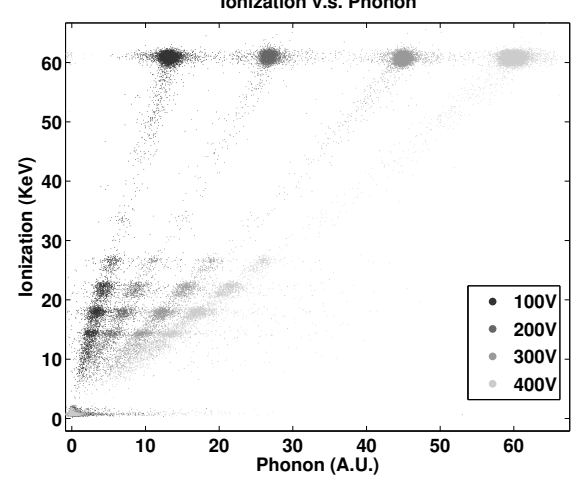

Figure 1: (Top Left) Schematic of the PPC detector used in this work. The point contact is defined by a $3 \mathrm{~mm}$ diameter vapor-deposited aluminum disk on the amorphous silicon passivation layer. The face opposite of the point contact and the cylindrical surface have lithium diffused to a depth of $\sim 1 \mathrm{~mm}$ coated with sputtered amorphous Ge followed by vapor-deposited aluminum. (Top right) Photo of the detector in its copper housing. The long wire bonds connect the point contact and the TES thermistors to the CDMS Digital Interface Board and corresponding cold electronics. (Bottom) Ionization versus phonon measurement for various bias voltages up to $400 \mathrm{~V}$. The phonon signal increases proportional to bias while ionization signal remains constant as expected. The $12 \%$ larger phonon gain for the $300 \mathrm{~V}$ and the $400 \mathrm{~V}$ data is a result of a jump in our readout SQUID circuit lock point that occurred at the end of the $200 \mathrm{~V}$ data acquisition.

is a linear function of applied voltage up to our limited DC voltage supply circuit.

The CDMS iZIP interface structure is symmetric (amorphous-Si only), in contrast with LBNL PPC design where the positive and negative bias electrodes are interfaced with different material (amorphous-Si or amorphous-Ge) to the bulk (Figure 1 Top left). A previous study by the LBNL group showed that the blocking properties of amorphous-Si or amorphous-Ge are different for different types of carriers and that amorphous-Ge better blocks holes than amorphous-Si [9]. However, these detectors are operated and tested at $>77 \mathrm{~K}$, well above freeze-out of Ge, and high voltage operations at the low temperatures needed for phonon sensing may require different contacts. 


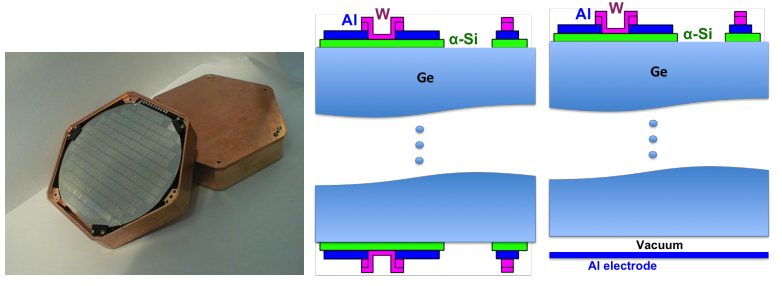

Figure 2: (Left) The CDMS ZIP phonon readout consist of $1024 \mathrm{~W}$ TES athermal phonon sensors covering one face of a $\varphi=75 \mathrm{~mm} \mathrm{~h}=10 \mathrm{~mm}$ Ge crystal. (Middle) The CDMS detector contact geometry is symmetric on both faces. The aluminum bias contacts are interfaced through a layer of $50 \mathrm{~nm} \alpha$-Si to the Ge crystal (Right) One face of the detector studied in this work is not processed (bare polished Ge) and the crystal is biased via a $\sim 500$ micron gap.

\subsection{New Contact Geometry}

To break CDMS design symmetry, we fabricated a detector for which we eliminate one interface and bias the detector with a flat aluminum electrode separated from the crystal via a small gap $\sim 500 \mu \mathrm{m}$ (Fig. 2). One face of a $0.250 \mathrm{~kg}$ Ge substrate was processed with athermal phonon sensors similar to CDMS ZIP detectors and was covered with athermal phonon tungsten (W) Transition Edge Sensors (TES). The phonon sensors also acted as the ground for biasing purposes and were interfaced to the detector through a layer of $\alpha$-Si. The other face of the detector was left with bare polished Ge. The detector was biased via the $\sim 500 \mu \mathrm{m}$ gap. The bias electrode was beveled to avoid the high fields associated with the sharp features at the edges of the electrodes. Because no carrier injection was expected from the contact-free face of the detector, this new biasing scheme allows for independent study of the $\mathrm{Al} / \alpha-\mathrm{Si} / \mathrm{Ge}$ interface properties.

The detector was mounted with a collimated ${ }^{241} \mathrm{Am}$ source on the phonon face and was installed in the Berkeley ${ }^{3} \mathrm{He}-{ }^{4} \mathrm{He}$ dilution refrigerator and cooled to $<0.04 \mathrm{~K}$. We discuss results from high voltage operation of this detector. A Light Emitting Diode (LED) with photon energies slightly above the Ge band gap was mounted close to the detector volume. By grounding the detector and pulsing the LED, large number of electrons and holes are generated to neutralize and reset the detector. Since the charge carriers that arrive to the bare face of the Ge have no path to leave the crystal, this LED pulsing is crucial to eliminate the accumulated charge and the counter electric field which results.

\section{Experimental results and discussion}

Leakage current in the new contact-free geometry for both positive and negative bias polarities was measured. The phonon surface was always held at the ground potential. All the bias polarities mentioned in this paper are referenced to the phonon face electrode. Therefore, for the positive bias polarities the holes drift toward the phonon surface and electrons toward the bias electrode and vice versa. Since there is only one contact present in this detector design (the phonon surface), we can study the polarity dependence of current leakage through $\mathrm{Al} /$ amorphous-Si/Ge with this device.

We use phonon noise performance as a metric to evaluate leakage. The carriers that are randomly leaking through the Ge electrode interface will also drift within the crystal volume producing NeganovLuke phonons and will appear as an increase in phonon readout noise. We found this method of leakage current measurement much more sensitive than the standard ionization direct charge readout.

In clear agreement with the previous studies with PPC detectors, we observed a leakage asymmetry with respect to the bias polarity in this device. The detector sustains up to $350 \mathrm{~V}$ in positive polarity but exhibits significant leakage for negative polarity even for biases as low as $20 \mathrm{~V}$ as shown in Fig. 3. Also in agreement with the previous LBNL results, this suggests that the amorphous-Si interface is a much weaker blocking layer for hole injection from $\mathrm{Al}$ compared to electron injection from the same.

Since the detector is biased via a gap of $\sim 500 \mu \mathrm{m}$, the actual potential across the detector $\left(\mathrm{V}_{G e}\right)$ is smaller than the applied voltage $\left(\mathrm{V}_{\text {applied }}\right)$ by the ratio of detector capacitance $(100 \mathrm{pF})$ and the gap. We use the phonon amplification gain for the ${ }^{241} \mathrm{Am}$ $13.9 \mathrm{keV}$ line compared to $\mathrm{V}=0 \mathrm{~V}$ data to estimate the true voltage across the detector. We found the $\mathrm{V}_{\text {Ge }} / \mathrm{V}_{\text {applied }}$ ratio to be $\sim 0.7$. We also confirmed Neganov-Luke gain linearity with respect to applied voltages up to $350 \mathrm{~V}$ (corresponding to $\mathrm{V}_{G e}=250 \mathrm{~V}$ ) as shown in Fig. 3. We observed slight noise increase associated with the rise of leakage current in the detector for $\mathrm{V}_{G e} \sim 140 \mathrm{~V}$ across the crystal. Assuming an almost uniform electric field in this geometry, this corresponds to a field of $\sim 140 \mathrm{~V} / \mathrm{cm}$ within the crystal volume.

RMS resolution was measured for every applied bias voltage by fitting phonon templates generated from ${ }^{241} \mathrm{Am} 13.9 \mathrm{keV}$ events to randomly triggered baseline traces. We fit a gaussian function to the re- 

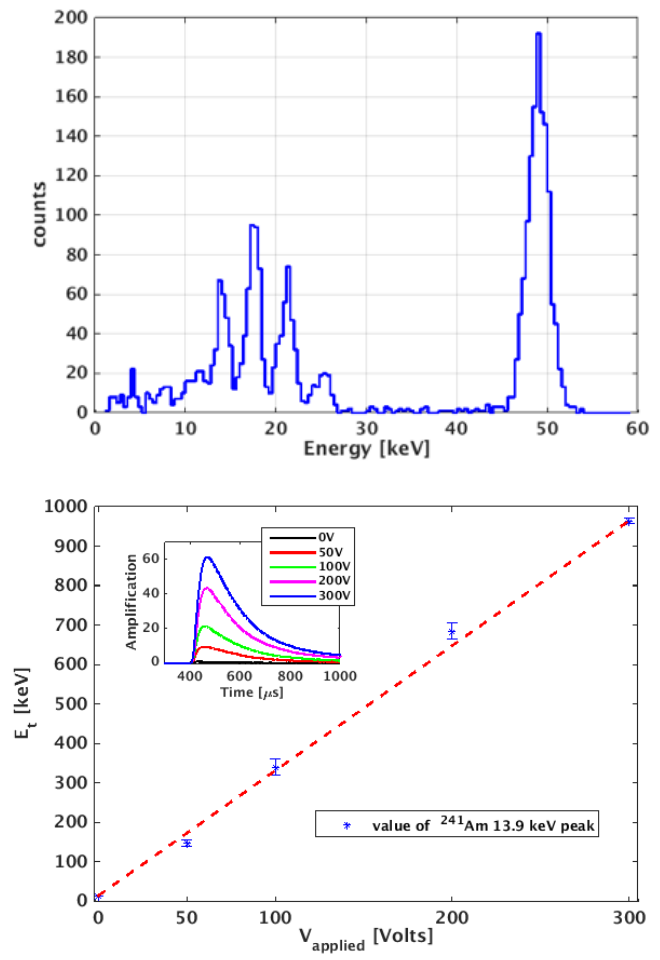

Figure 3: Top) Spectrum of ${ }^{241} \mathrm{Am}$ source obtained at $\mathrm{V}_{\text {bias }}=300 \mathrm{~V}$. The $60 \mathrm{keV}$ photon line appears at lower energy due to large phonon gain and TES saturation. Bottom) Linear phonon gain and phonon pulses for the $13.9 \mathrm{keV}$ line as a function of bias. The pulse amplitudes increase linearly with bias as expected. We can use the line fit to estimate the vacuum gap between the bias electrode and the crystal as described in the text.
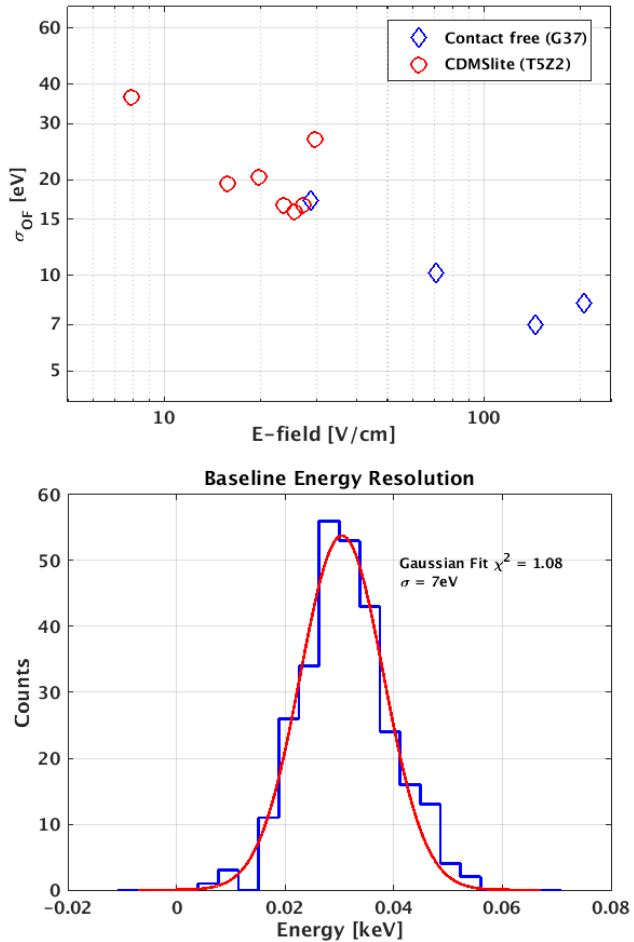

Figure 4: Top) Ionization base line RMS resolution as a function of E-field for CDMSlite [10] (red circles) and the contact-free detector reported in this work. A clear sign of leakage is observed for fields above $24 \mathrm{~V} / \mathrm{cm}$ in CDMSlite detectors. The contact-free detector RMS resolution continues to improve for $\mathrm{E}>100 \mathrm{~V} / \mathrm{cm}$. Bottom) The best RMS resolution measured in this geometry was slightly above $7 \mathrm{eV}$. The energy distribution is not zero-centered because the fitting algorithm works by searching for the largest positive noise excursion. The same optimal filter is used to fit the (positive) phonon pulses from regular events. The phonon gain at constant electric field scales linearly with thickness, thus we expect to have $<3 \mathrm{eV}$ resolution using a CDMSlite detector geometry. 
sulting distribution and use this fit to calculate the $\sigma$ of the distribution. In Fig. 4 we compare phonon resolution progression with respect to the applied electric field between CDMSlite and contact-free geometries. As expected, the resolution improves linearly with bias up until the detector exhibits significant leakage, thus increased phonon noise. The leakage onset clearly occurs at much smaller fields in CDMSlite $(\sim 25 \mathrm{~V} / \mathrm{cm})$ than contact free. The best RMS resolution obtained with this contact-free prototype detector (corresponding to $\mathrm{V}_{G e} \sim 140 \mathrm{~V}$ ) was slightly below $7 \mathrm{eV}$. This is the world best ionization baseline resolution for a detector of this size.

The prototype contact-free detector presented here is $1 \mathrm{~cm}$ thick (i.e. $\times 2.5$ thinner than CDMSlite detectors). Since the leakage current is a function of the electric field and the phonon gain is a function of potential, we expect a straightforward improvement of $\times 2.5$ in phonon resolution down to $\sigma=2.8$ $\mathrm{eV}$ using the contact-free biasing scheme on $2.5 \mathrm{~cm}$ thick CDMSlite geometry. Our detectors are sensitive to athermal phonons, thus the larger volume of the detector should not affect phonon collection efficiency since a majority of the phonons will relax in the metallic sensors.

In order to reach better resolutions, the interface device physics must be adapted to maintain low hole and electron injection from the $\mathrm{Al}$ while allowing phonon transfer to the TES. We propose that a dielectric with significant electron and hole band edge offsets, but sufficiently thin for phonon transparency, is the best option. However, formation of quality dielectrics at low temperatures so as to not create di-vacancies and other charge trapping defects is a critical challenge. We are currently investigating $\mathrm{SiO}_{2}$, sapphire and nitrides for just such an application.

\section{Conclusion}

Combined results from prior tests of LBNL PPC and our new contact-free detectors point to the specific CDMSlite contact design to be the source for low field leakage current observed in CDMS. We found that $\mathrm{Al} / \alpha$-Si/Ge has asymmetric blocking properties for electron versus hole injection and provides better electron blockage. Our results are in agreement with previous LBNL results wherein amorphous Ge had beed suggested to block hole injection. CDMS is currently studying $\alpha$-Ge and other dielectric material as alternatives for a contact interface.
Our new contact-free detector can sustain substantially larger bias than CDMSlite detectors. Using the Neganov-Luke phonon amplification method, we achieved world best RMS resolution of $7 \mathrm{eV}$ with this prototype detector. A further $\times 2.5$ gain in resolution (to $\sigma=2.8 \mathrm{eV}$ ) is readily available by scaling the detector thickness to that of CDMSlite detectors. However, in order to reach resolutions as low as single electrons, we will need to fully isolate the phonon readout surface of the detector. One possibility is to use a thin layer of $\mathrm{SiO}_{2}$ to interface the phonon readout surface to the detector. It has been recently suggested that operating detectors with lower $\mathrm{T}_{c}$ phonon sensors will improve resolution to the $\mathrm{eV}$ scale [11]. The combination of this work and those studies can lead to the ultimate single electron resolution detectors with event-by-event discrimination capability.

\section{Acknowledgments}

This work has been supported in part by the National Science Foundation under the awards PHY - 1102841 and PHY - 1242645 and by the University of California MRPI Search For Dark Matter Initiative and DOE grant DE-SC0004022. The authors wish to acknowledge CDMS collaboration support and very useful discussions with Paul Luke, Kai Vetter, Mark Amman, Ryan Martin, Paul Brink, Matt Pyle, Rito Basu Thakur. This work wouldn't be possible without technical support form Mark Platt, James Phillips and Matthew Cherry.

\section{References}

[1] P. A. R. Ade, et al., Planck 2013 results. XVI. Cosmological parameters (2013). arXiv:arXiv:1303.5076.

[2] P. Cushman, C. Galbiati, D. N. McKinsey, H. Robertson, T. M. P. Tait, D. A. Bauer, A. Borgland, B. Cabrera, F. Calaprice, J. Cooley, et al., Snowmass CF1 summary: WIMP dark matter direct detection (2013). arXiv:arXiv:1310.8327.

[3] D. Z. Freedman, Coherent neutrino nucleus scattering as a probe of the weak neutral current, Phys. Rev. D9 (1974) 1389-1392.

[4] B. Dutta, R. Mahapatra, L. Strigari, J. Walker, Sensitivity to Z-prime and non-standard neutrino interactions from ultra-low threshold neutrino-nucleus coherent scattering (2015). arXiv:ArXiv:1508.07981.

[5] J. A. Formaggio, E. Figueroa-Feliciano, A. J. Anderson, Sterile neutrinos, coherent scattering and oscillometry measurements with low-temperature bolometers (2011). arXiv:arXiv:1107.3512. 
[6] P. N. Luke, J. Beeman, F. S. Goulding, S. E. Labov, E. H. Silver, Calorimetric ionization detector, Nucl. Instrum. Meth. A289 (1990) 406-409.

[7] R. Agnese, et al., CDMSlite: A Search for low-mass WIMPs using voltage-assisted calorimetric ionization detection in the superCDMS experiment, Phys. Rev. Lett. 112 (2014) 041302.

[8] N. Mirabolfathi, J. J. Yen, P. L. Brink, B. Cabrera et al., Contact-free germanium ionization and phonon detectors, in: G. T. Rado, H. Suhl (Eds.), AIP Conf. Proc., Vol. 1185, 2009, pp. 647-650.

[9] M. Amman, P. N. Luke, S. E. Boggs, Amorphoussemiconductor-contact germanium-based detectors for gamma-ray imaging and spectroscopy, Nucl. Instrum. Meth. A579 (2007) 886-890.

[10] R. B. Thakur, PhD dissertation, University of Illinois at Urbana-Champaign (2015).

11] M. Pyle, E. Figueroa-Feliciano, B. Sadoulet, Optimized designs for very low temperature massive calorimeters (2015). arXiv:arXiv:1503.01200. 